\title{
Lipopolysaccharide Animal Models for Parkinson's Disease
}

\author{
Mei Liu and Guoying Bing \\ Department of Anatomy and Neurobiology, College of Medicine, University of Kentucky, Lexington, KY 40536, USA
}

Correspondence should be addressed to Guoying Bing, gbing@uky.edu

Received 24 November 2010; Accepted 28 February 2011

Academic Editor: Gilles J. Guillemin

Copyright () 2011 M. Liu and G. Bing. This is an open access article distributed under the Creative Commons Attribution License, which permits unrestricted use, distribution, and reproduction in any medium, provided the original work is properly cited.

\begin{abstract}
Lipopolysaccharide (LPS), an endotoxin from Gram-negative bacteria, acts as a potent stimulator of microglia and has been used to study the inflammatory process in the pathogenesis of Parkinson's disease (PD) and anti-inflammatory therapy for PD treatment. Here, we review the growing body of literature on both in vitro and in vivo LPS PD models. Primary cell cultures from mesencephalic tissue were exposed to LPS in vitro; LPS was stereotaxically injected into the substantia nigra, striatum, or globus pallidus of brain or injected into the peritoneal cavity of the animal in vivo. In conclusion, the LPS PD models are summarized as (1) local and direct LPS treatment and (2) systemic LPS treatment. Mechanisms underlying the PD models are investigated and indicated that LPS induces microglial activation to release a variety of neurotoxic factors, and damaged neurons may trigger reactive microgliosis, which lead to progressive dopaminergic neurodegeneration.
\end{abstract}

\section{Introduction}

Parkinson's disease (PD) is the most prevalent neurodegenerative movement disorder. In $\mathrm{PD}$, clinical symptoms including tremor, rigidity, and bradykinesia are primarily resulted from the loss of dopamine-containing neurons in the substantia nigra pars compacta. Although the etiology and pathogenesis of PD remain not fully elucidated, many interacting pathological processes appear to contribute to dopaminergic neuron degeneration in the disease. Recently, inflammatory processes have been implicated as one of the active contributors to dopaminergic neuron damage in the development and progression of the disease [1, 2]. In the central nervous system, microglia, the resident innate immune cells, play a major role in the inflammatory process. Typically microglia exist in a resting state characterized by ramified morphology and monitor the brain environment [3]. In response to various pathogenic stimuli including inflammation, microglia are readily activated and undergo a transformation to amoeboid morphology with an upregulated catalogue of surface molecules [3-5]. Activated microglia can serve diverse beneficial functions essential to neuron survival, which include cellular maintenance and innate immunity [6]. However, uncontrolled activated microglia produces a variety of neurotoxic factors such as proinflammatory cytokines (interleukin-1 (IL-1), tumor necrosis factor alpha (TNF- $\alpha$ ), interleukin-6 (IL-6)), nitro oxide (NO), prostaglandin E2, and superoxide, which lead to neuronal damage or death [1,7-10]. Additionally, damaged neurons may emit injury signals to cause microglia activation, which used to be defined as reactive microgliosis [11]. This microglial-neuronal interaction will be reinforced and become a self-amplifying cycle of neuronal injury and microglial activation, which finally leads to more neuronal damage and death. Importantly, clinical researches have reported that microglial activation was found in the nigrostriatal system of PD patients [12-14]. Therefore, it is essential to study the inflammatory process in $\mathrm{PD}$, which may help us understand the pathogenesis of the disease and eventually develop an effective therapeutic strategy.

Over the last two decades, studies in animal models have demonstrated that inflammation induced by lipopolysaccharide (LPS) can replicate some characteristics of PD, including extensive activation of microglia and selective loss of dopaminergic neurons in the nigrostriatal system [15-19]. The history of understanding LPS starts in the late nineteenth century. LPS is found in the outer membrane of Gramnegative bacteria and acts as endotoxin. LPS from many Gram-negative bacteria species initiates acute inflammatory responses in mammals and induces a diverse range of effects, ranging from pyrexia to Gram-negative septic shock [20]. Thus, using different serotypes of LPS and their different 
application routes may cause different outcomes [21]. Moreover, LPSs from different bacteria species share common features in their basic architecture, which consists of three covalently linked segments, a surface carbohydrate polymer (Ospecific chain), a core oligosaccharide featuring an outer and inner region, and an acylated glycolipid (termed lipid A). The $\mathrm{O}$-specific chain shows the most diversity and is the basis for serological specificity, while lipid A, which anchors the LPS molecule in the Gram-negative outer membrane, is the most conserved biochemical structure across different bacterial species [20]. There is wide acceptance that the lipid A moiety is the innate immune stimulating or endotoxic component of LPS [22]. In addition, it is documented that LPS-associated pathology results from the stimulation of host cell responses, in which LPS binds to specific receptors in order to elicit the release of cytokines and other inflammatory mediators. Several membrane-bound and soluble proteins have been shown to bind LPS; the most important appear to be CD14 and LPSbinding protein (LBP) and the toll-like receptor (TLR) family which is a recently discovered group of transmembrane receptors [23, 24]. In the central nervous system, it is found that systemic LPS injection upregulated its membrane CD14 receptor within specific cellular populations including microglia in the brain [25]. Thereafter, microglia were identified as the major LPS-responsive cell in the brain. LPS binds to TLR4 on microglia and induces microglial activation that results in neuronal damage $[26,27]$.

LPS acts as an endotoxin and elicits multiple pathological effects in human beings. One case report may uncover a potential link between LPS infection and the development of Parkinsonism. A 22-year-old laboratory worker was accidentally exposed to $10 \mu \mathrm{g}$ Salmonella minnesota LPS through an open wound and developed Parkinson's syndrome with bradykinesia, rigidity, tremor, and cogwheel phenomenon three weeks later; damage to the substantia nigra and cerebral cortex was shown by positron emission tomography a few years after the accident [28]. However, it is known that LPS from many bacterial species such as Salmonella, Pseudomonas, Vibrio, and Rhizobium can initiate acute inflammatory responses in mammals and induce a large and diverse range of effects, ranging from pyrexia and Gramnegative septic shock [29]. There is another case report regarding the Salmonella endotoxin exposure. One middleaged laboratory worker was self-administered intravenously a single large dose of endotoxin (1 mg Salmonella minnesota LPS) and immediately developed a severe septic shock syndrome with multiple-organ dysfunction. The patient was successfully rescued in the emergency room, and there has been no follow-up report to date [30]. Thus, further investigation and more epidemiologic data are needed to exploit the relationship between endotoxin and PD.

In the current paper, we present a summary of a variety of LPS PD models and discuss their strengths and limitations, which may be helpful for the future LPS PD study.

\section{In Vitro Studies of LPS PD Model}

2.1. LPS Treatment to Cell Culture from Mesencephalic Tissue. Bronstein et al. in 1995 reported the comparison study between the dopaminergic neurotoxin 6-hydroxydopamine (6-OHDA) and LPS in rat mesencephalic cultures [31]. Investigators found that, in the neuron-enriched cultures, 6-OHDA killed $89 \%$ of the tyrosine hydroxylase- (TH-) immunopositive neurons, but LPS $(50 \mu \mathrm{g} / \mathrm{mL})$ was not neurotoxic; however, in the mixed neuron-glial cultures, 6OHDA killed only $27 \%$ of the TH-immunopositive neurons, but LPS killed $70 \%$ of the TH-immunopositive neurons. This early experiment suggested that the dopaminergic neurotoxicity of LPS is dependent on the presence of microglia. Subsequently, dopaminergic neurotoxicity of LPS was confirmed by the other groups on rat mesencephalic mixed neuron-glial cultures and demonstrated that LPS induced microglial activation, and activated microglia released the proinflammatory and cytotoxic factors: NO, TNF- $\alpha$, and IL$1 \beta$, which lead to dopaminergic neuron damage $[32,33]$. In addition, the dopaminergic neurotoxicity of LPS was studied on mouse mesencephalic neuron-glial culture, and it was found that the neurotoxicity was mainly mediated through LPS-induced nicotinamide adenine dinucleotide phosphate (NADPH) oxidase activation on microglia, which generated reactive oxygen species production, which are neurotoxic factors [34].

In the studies of LPS-treated primary cultures generated from forebrains of embryonic day 17 mice, the investigators found that the LPS neurotoxicity occurred through binding the signal-transducing receptor, TLR4; microglia are the major cells in the central nervous system that express TLR4. However, it is emphasized that the toxic effect of LPS on neurons is a general phenomenon, independent of neuronal subtype [26]. Thus, LPS may cause neurotoxicity without selectivity for neuronal types. Since previous studies from primary microglia cultures have found LPS treatment induced the release of proinflammatory and cytotoxic factors from microglia $[35,36]$, the investigators suggested that the activation of TLR4 on microglia may initiate the intracellular signaling pathway of microglia, and result in the release of proinflammatory mediators which cause neuronal damage [26].

\section{In Vivo Studies of LPS PD Model}

3.1. Intranigral Injection of LPS. In order to study the response of the nigrostriatal system to inflammation, Bing et al. and Castaño et al. independently reported the PD model of LPS intranigral injection in 1998 [15, 16]. After LPS was stereotaxically injected into the nigral area of rats, investigators found that LPS induced microglia activation and dopaminergic neuron loss in the substantia nigra [15, 16]. In a following study, it was reported that LPS-induced dopaminergic neuronal damage was permanent, as observed one year postinjection. Moreover, there was no detectable damage to either the GABAergic or the serotoninergic neurons in the striatum and nigra after LPS injection, indicating that LPS selectively induced dopaminergic neuron death in the nigrostriatal system [37]. Thereafter, more studies confirmed the results and also found the increased level of proinflammatory cytokines including IL- $1 \beta$, TNF$\alpha$, IL- 6 , and NO in the substantia nigra after LPS injection, 
which may be causal factor for LPS-induced neuronal damage [38-40]. In addition, the effects of intranigral LPS injection on behavior and dopamine content and turnover were investigated and showed that LPS treatment enhanced locomotor activity 2- to 3-fold and increased dopamine turnover ratios in comparison with control subjects. This suggests that LPS insult may induce a compensatory response of dopaminergic system [41].

3.2. Intrapallidal Injection of LPS. The globus pallidus is a major integrative nucleus within the basal ganglia, with neurons projecting to striatum, subthalamic nucleus, entopeduncular nucleus, and substantia nigra. Thus, the globus pallidus is positioned to influence the nigrostriatal pathway and function of the basal ganglia as a whole. LPS was injected into the globus pallidus of young and middle-aged rats. The results showed that microglial activation was found in both globus pallidus and substantia nigra, dopaminergic neurons were significantly and progressively decreased in the substantia nigra, and locomotor deficits were detected in animal after LPS injection [17]. Moreover, the following study reported an increased level of proinflammatory cytokines including IL- $1 \beta$, TNF- $\alpha$, and IL- 6 , the elevated expression of inducible nitric oxide synthase, and the enhanced $\alpha$-synuclein nitration and oligomerization in the substantia nigra of LPS-injected animal [42]. Interestingly, the above pathological changes were much severer in middle-aged animals when compared with the younger animals after LPS treatment, supporting the view that aging itself is a risk factor for PD development [42]. Inflammation promotes the release of neurotoxic factors and the development of synucleinopathy lesions that finally lead to dopaminergic neurodegeneration in PD model of LPS intrapallidal injection. Additionally, the finding of abnormal $\alpha$-synuclein may help us to explore the mechanisms underlying progressive loss of dopaminergic neurons in LPS PD models. It is reported that aggregated $\alpha$-synuclein induced microglial activation in a primary mesencephalic neuron-glia culture system [43], thus the pathological process of reactive microgliosis may be triggered and microglial activation may become uncontrolled, which eventually result in progressive dopaminergic neurotoxicity.

3.3. Intrastriatal Injection of LPS. In the nigrostriatal system, the cell bodies of dopaminergic neurons are located in the substantia nigra and their dopamine-containing terminals are distributed in the striatum. After LPS was injected into the striatum of rats, we detected a progressive degeneration of dopamine cell bodies in the substantia nigra and their axonal terminals in the striatum, a depletion of dopamine content in the striatum, cytoplasmic accumulation of $\alpha$ synuclein and ubiquitin in the nigral dopamine neurons, and behavioral deficits assessed by cylinder test and amphetamine-induced rotational behavior behavioral test [19, 44-46]. Molecular mechanisms underlying the neurotoxicity of LPS intrastriatal injection included activation of microglia, impairment of mitochondria state III and state $\mathrm{V}$ respiration, and an increased release of proinflammatory mediators: IL- $1 \beta$, TNF- $\alpha$, IL- 6 , IL- $1 \alpha$, and NO, in both the substantia nigra and the striatum. This indicates that the inflammatory insult or stimuli in the striatum not only directly damaged the terminals of dopaminergic neurons in the striatum, but also indirectly damaged the cell bodies of dopaminergic neurons in the substantia nigra through an unknown retrograde signal transduction pathway $[19,44$, $45]$.

3.4. Intraperitoneal/Systemic Injection of LPS. To study how infectious disease through blood transmission affects the development of neurodegenerative disease in the central nervous system, LPS was systemically injected into animals. Early work reported that after systemic (intraperitoneal or intravenous) injection of LPS, LPS has the ability to target the brain in upregulating its membrane CD14 receptor within specific cellular populations including microglia, which is likely to be responsible for the transcription of proinflammatory cytokines: first within accessible structures from the blood and thereafter through scattered parenchymal cells during severe sepsis [25]. In addition, early work also showed that intraperitoneal endotoxin even at a high dose $(2 \mathrm{mg} / \mathrm{kg}$ of LPS, which has cardiovascular effects, e.g., a decrease in blood pressure) into rats did not disrupt blood-brain barrier (BBB) permeability, suggesting that intraperitoneal LPS administration is unlikely to contribute to the observed central nervous system mediated effects of endotoxin [47]. However, other studies have found that some cytokines including TNF- $\alpha$ and IL-1 can be transported across the BBB by saturable transport systems, which are able to directly affect central nervous system functions [48, 49]. Using the intraperitoneal injection of LPS in mice, Qin et al. reported that increased cytokine TNF- $\alpha$ due to LPS insult was critical for the transfer of inflammation from the periphery to the central nervous system to induce microglial activation and dopaminergic neuron loss in the substantia nigra at 7 and 9 months posttreatment [18]. Nevertheless, Byler et al. found that systemic LPS injection alone did not affect dopamine levels or Parkinsonian behavioral tests in mice whereas systemic LPS plus MPTP in combination induced the depletion of dopamine in the striatum and Parkinsonian behavioral deficits (reduced stride length) at 4 months postinjection [50]. In addition, MPTP treatment alone reduced striatal dopamine levels quickly but they recovered to normal levels later, addressing the point that nigrostriatal dopamine neurons may succumb after time to multiple toxic agents [50].

\section{Implication Using the Animal LPS PD Models}

A number of studies have suggested that microglial activation plays a key role in the initiation and progression of $\mathrm{PD}$ $[8,12,13,51,52]$. LPS PD models provide us with a good tool to investigate the inflammatory process in PD development and anti-inflammation therapy for PD treatment. For example, naloxone, an antagonist of opioid receptors, provided the dopaminergic neuroprotective effects against LPS damage $[32,38,53]$. Interleukin-10, a natural immune modulator, reduced LPS-induced dopaminergic neurotoxicity by inhibiting microglial activation $[40,54]$. Pioglitazone, an agonist of peroxisome proliferator-activated 


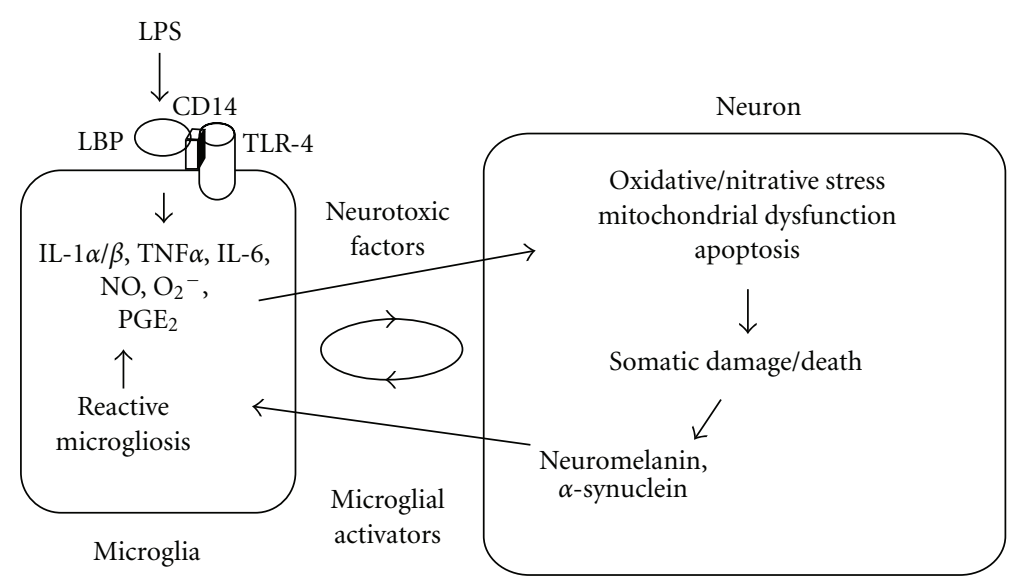

FIGURE 1: LPS induces progressive neurotoxicity. In response to LPS stimuli, microglial cells are readily activated. It is demonstrated that LPS binds to specific receptors, for example, CD14/TLR4/LBP receptor complex on the microglia, to induce microglial activation. Uncontrolled microglial activation produce a variety of neurotoxic factors such as proinflammatory cytokines (IL-1, TNF- $\alpha$, IL-6), NO, $\mathrm{PGE}_{2}$, and $\mathrm{O}_{2}{ }^{-}$, which lead to neuronal damage or death through a cascade of events such as oxidative/nitrative stress, mitochondrial dysfunction, and apoptosis. Moreover, damaged neurons may emit injury signals to cause microglia activation, which is defined as reactive microgliosis. The injury signals could be neuromelanin and $\alpha$-synuclein released by injured dopaminergic neurons. This microglial-neuronal interaction will be reinforced and become a self-amplifying cycle of neuronal injury and microglial activation, which may finally result in the neurodegenerative disease.

receptor gamma, improved dopaminergic neuron survival by restoring mitochondrial function, decreasing the release of proinflammatory mediators and suppressing the oxidative stress $[33,45,55,56]$. Minocycline, a semisynthetic second-generation tetracycline, exerts potential neuroprotective effects by reducing the inflammatory response and inhibiting apoptotic cell death [57-59]. Among all of these, minocycline is receiving a great deal of attention for its potent antiinflammatory and anti-apoptosis effects. It has been demonstrated that minocycline has few safety concerns and that it should be considered for a large phase III efficacy trial after phase II clinical trials in early PD patients [60, 61]. Currently, minocycline is used in many ongoing clinical trials for various diseases including PD [62].

\section{Discussion of LPS PD Models}

Mechanisms underlying the LPS PD models are investigated and indicated that LPS induces microglial activation, activated microglia release proinflammatory and neurotoxic factors such as IL- 1 , TNF- $\alpha$, IL- 6 , and NO to cause neuronal damage $[40,42,63]$, and damaged neuron may emit injury signals such as neuromelanin and abnormal $\alpha$-synuclein to trigger reactive microgliosis $[43,64,65]$. This neuronalmicroglial interaction may be reinforced and become a selfamplifying cycle to result in progressive dopaminergic neurodegeneration (Figure 1). Based on the application routes in these LPS PD models, we summarize them as follows: (1) LPS is directly and locally applied into the nigrostriatal system and its related structures, such as LPS treatment in mesencephalic cell culture systems in vitro and stereotaxic injection of LPS in nigra, striatum or globus pallidus in vivo; (2) LPS is systemically administered and selectively affects the nigrostriatal system, such as intraperitoneal injection of
LPS in vivo. First, let us discuss the local and direct LPS treatment of PD models. Many studies have suggested that dopaminergic neurons are more vulnerable than others in the nigrostriatal system to inflammation-mediated neurotoxicity owing to their precarious redox equilibrium and colocalization with a large population of microglia $[2,66]$. Thus, inflammatory responses induced by direct and local LPS treatment may selectively cause dopaminergic neuron damage in mesencephalic tissue in vitro and in the nigrostriatal system in vivo. For stereotaxic injection of LPS in nigra, striatum, or globus pallidus in vivo, because of the smaller size of the nigral area compared with the striatum/globus pallidus area and the dense distribution of dopaminergic neurons in the nigra, intranigral injection itself may cause severe mechanical injury to neurons and glial cells in the nigral area whereas intrastriatal/intrapallidal LPS injection has the advantage of keeping intact the structure of the nigra for enabling the study of the toxic effect of inflammation on neurons. Moreover, intrastriatal/intrapallidal LPS treatment not only induces progressive dopaminergic neuron loss, but also leads to behavioral deficits in animal studies. Thus intrastriatal/intrapallidal LPS injection may be a better PD model in vivo. Next, let us discuss the systemic LPS treatment of PD model. There remains a puzzle how systemic treatment of LPS selectively induced dopaminergic neuron death in the nigrostriatal system of brain. We know that LPS acts as a potent stimulator of microglia and microglia density varies by brain region in human and animals. It has been reported that the level of microglial cells was high in the medulla oblongata and pons in comparison with that in the substantia nigra, hippocampus, thalamus, basal ganglia, and pedunculus cerebri in an adult normal human brain study [67]. Likewise, microglial cells are not uniformly distributed in the normal adult mouse brain. Lawson et al. 
reported that microglial densely populated areas include the hippocampus, olfactory telencephalon, basal ganglia, and substantia nigra in the adult mouse brain [68]. Importantly, these studies demonstrate that the density of microglial cells in the substantia nigra is similar to that in the hippocampus, basal ganglia, and so on for both the human and mouse brains. In other words, microglia activation and subsequent proinflammatory cytokines release due to LPS insult may occur in several brain regions, but not in nigral area alone. For example, LPS is also widely used in experimental in vitro and in vivo models of inflammation and amyloidosis for Alzheimer's disease [69, 70]. Thus, it needs further investigation for the selective dopaminergic neurodegeneration in the substantia nigra after systemic LPS treatment. In summary, bacterial endotoxin LPS used as a potent stimulator of glial cells, especially microglia, help us to study the molecular mechanism underlying inflammatory processes in neurodegenerative diseases in the central nervous system. Direct and local LPS treatment in the nigrostriatal system and its related structures may be better PD models to study the etiology and therapeutic strategies for inflammation in PD.

\section{Acknowledgment}

The authors greatly thank Dr. Wayne A. Cass for the helpful comments and suggestions.

\section{References}

[1] Y. S. Kim and T. H. Joh, "Microglia, major player in the brain inflammation: their roles in the pathogenesis of Parkinson's disease," Experimental and Molecular Medicine, vol. 38, no. 4, pp. 333-347, 2006.

[2] M. L. Block, L. Zecca, and J. S. Hong, "Microglia-mediated neurotoxicity: uncovering the molecular mechanisms," Nature Reviews Neuroscience, vol. 8, no. 1, pp. 57-69, 2007.

[3] A. Nimmerjahn, F. Kirchhoff, and F. Helmchen, "Neuroscience: resting microglial cells are highly dynamic surveillants of brain parenchyma in vivo," Science, vol. 308, no. 5726, pp. 1314-1318, 2005.

[4] D. Davalos, J. Grutzendler, G. Yang et al., "ATP mediates rapid microglial response to local brain injury in vivo," Nature Neuroscience, vol. 8, no. 6, pp. 752-758, 2005.

[5] B. P. Cho, D. Y. Song, S. Sugama et al., "Pathological dynamics of activated microglia following medial forebrain bundle transection," Glia, vol. 53, no. 1, pp. 92-102, 2006.

[6] W. J. Streit, "Microglia as neuroprotective, immunocompetent cells of the CNS," Glia, vol. 40, no. 2, pp. 133-139, 2002.

[7] R. B. Banati, S. E. Daniel, and S. B. Blunt, "Glial pathology but absence of apoptotic nigral neurons in long-standing Parkinson's disease," Movement Disorders, vol. 13, no. 2, pp. 221-227, 1998.

[8] J. W. Langston, L. S. Forno, J. Tetrud, A. G. Reeves, J. A. Kaplan, and D. Karluk, "Evidence of active nerve cell degeneration in the substantia nigra of humans years after 1-methyl-4phenyl-1,2,3,6-tetrahydropyridine exposure," Annals of Neurology, vol. 46, no. 4, pp. 598-605, 1999.

[9] C. F. Orr, D. B. Rowe, Y. Mizuno, H. Mori, and G. M. Halliday, "A possible role for humoral immunity in the pathogenesis of Parkinson's disease," Brain, vol. 128, no. 11, pp. 2665-2674, 2005.
[10] M. L. Block and J. S. Hong, "Microglia and inflammationmediated neurodegeneration: multiple triggers with a common mechanism," Progress in Neurobiology, vol. 76, no. 2, pp. 77-98, 2005.

[11] W. J. Streit, S. A. Walter, and N. A. Pennell, "Reactive microgliosis," Progress in Neurobiology, vol. 57, no. 6, pp. 563$581,1999$.

[12] P. L. McGeer, S. Itagaki, H. Akiyama, and E. G. McGeer, "Rate of cell death in parkinsonism indicates active neuropathological process," Annals of Neurology, vol. 24, no. 4, pp. 574-576, 1988.

[13] P. L. McGeer, S. Itagaki, B. E. Boyes, and E. G. McGeer, "Reactive microglia are positive for HLA-DR in the substantia nigra of Parkinson's and Alzheimer's disease brains," Neurology, vol. 38, no. 8, pp. 1285-1291, 1988.

[14] K. Imamura, N. Hishikawa, M. Sawada, T. Nagatsu, M. Yoshida, and Y. Hashizume, "Distribution of major histocompatibility complex class II-positive microglia and cytokine profile of Parkinson's disease brains," Acta Neuropathologica, vol. 106, no. 6, pp. 518-526, 2003.

[15] G. Bing, X. Lu, N. A. Zheng, L. Jin, Y. Qi, and H.-C. Kim, "Microglia mediated dopaminergic cell death in the substantia nigra: a new animal model for Parkinson's disease," Neuroscience Abstracts, vol. 24, p. 44, 1998.

[16] A. Castaño, A. J. Herrera, J. Cano, and A. Machado, "Lipopolysaccharide intranigral injection induces inflammatory reaction and damage in nigrostriatal dopaminergic system," Journal of Neurochemistry, vol. 70, no. 4, pp. 15841592, 1998.

[17] J. Zhang, D. M. Stanton, X. V. Nguyen et al., "Intrapallidal lipopolysaccharide injection increases iron and ferritin levels in glia of the rat substantia nigra and induces locomotor deficits," Neuroscience, vol. 135, no. 3, pp. 829-838, 2005.

[18] L. Qin, X. Wu, M. L. Block et al., "Systemic LPS causes chronic neuroinflammation and progressive neurodegeneration," Glia, vol. 55, no. 5, pp. 453-462, 2007.

[19] D. Y. Choi, M. Liu, R. L. Hunter et al., "Striatal neuroinflammation promotes parkinsonism in rats," PLoS One, vol. 4, no. 5, Article ID e5482, 2009.

[20] J. Schletter, H. Heine, A. J. Ulmer, and E. T. Rietschel, "Molecular mechanisms of endotoxin activity," Archives of Microbiology, vol. 164, no. 6, pp. 383-389, 1995.

[21] T. Nedrebø and R. K. Reed, "Different serotypes of endotoxin (lipopolysaccharide) cause different increases in albumin extravasation in rats," Shock, vol. 18, no. 2, pp. 138-141, 2002.

[22] R. J. Ulevitch and P. S. Tobias, "Recognition of Gram-negative bacteria and endotoxin by the innate immune system," Current Opinion in Immunology, vol. 11, no. 1, pp. 19-22, 1999.

[23] K. Takeda, T. Kaisho, and S. Akira, "Toll-like receptors," Annual Review of Immunology, vol. 21, pp. 335-376, 2003.

[24] C. A. Janeway Jr. and R. Medzhitov, "Innate immune recognition," Annual Review of Immunology, vol. 20, pp. 197-216, 2002.

[25] S. Lacroix, D. Feinstein, and S. Rivest, "The bacterial endotoxin lipopolysaccharide has the ability to target the brain in upregulating its membrane CD14 receptor within specific cellular populations," Brain Pathology, vol. 8, no. 4, pp. 625640, 1998.

[26] S. Lehnardt, L. Massillon, P. Follett et al., "Activation of innate immunity in the CNS triggers neurodegeneration through a Toll-like receptor 4-dependent pathway," Proceedings of the National Academy of Sciences of the United States of America, vol. 100, no. 14, pp. 8514-8519, 2003. 
[27] K. Hoshino, O. Takeuchi, T. Kawai et al., "Cutting edge: Toll-like receptor 4 (TLR4)-deficient mice are hyporesponsive to lipopolysaccharide evidence for TLR4 as the Lps gene product," Journal of Immunology, vol. 162, no. 7, pp. 3749$3752,1999$.

[28] I. Niehaus and J. H. Lange, "Endotoxin: is it an environmental factor in the cause of Parkinson's disease?" Occupational and Environmental Medicine, vol. 60, no. 5, p. 378, 2003.

[29] I. Stewart, P. J. Schluter, and G. R. Shaw, "Cyanobacterial lipopolysaccharides and human health-a review," Environmental Health, vol. 5, article 7, 2006.

[30] A. M. T. da Silva, H. C. Kaulbach, F. S. Chuidian, D. R. Lambert, A. F. Suffredini, and R. L. Danner, "Brief report: shock and multiple-organ dysfunction after self-administration of salmonella endotoxin," The New England Journal of Medicine, vol. 328, no. 20, pp. 1457-1461, 1993.

[31] D. M. Bronstein, I. Perez-Otano, V. Sun et al., "Glia-dependent neurotoxicity and neuroprotection in mesencephalic cultures," Brain Research, vol. 704, no. 1, pp. 112-116, 1995.

[32] B. Liu, L. Du, and J. S. Hong, "Naloxone protects rat dopaminergic neurons against inflammatory damage through inhibition of microglia activation and superoxide generation," Journal of Pharmacology and Experimental Therapeutics, vol. 293, no. 2, pp. 607-617, 2000.

[33] B. Xing, T. Xin, R. L. Hunter, and G. Bing, "Pioglitazone inhibition of lipopolysaccharide-induced nitric oxide synthase is associated with altered activity of p38 MAP kinase and PI3K/ Akt," Journal of Neuroinflammation, vol. 5, article 4, 2008.

[34] L. Qin, Y. Liu, T. Wang et al., "NADPH oxidase mediates lipopolysaccharide-induced neurotoxicity and proinflammatory gene expression in activated microglia," Journal of Biological Chemistry, vol. 279, no. 2, pp. 1415-1421, 2004.

[35] S. C. Lee, W. Liu, D. W. Dickson, C. F. Brosnan, and J. W. Berman, "Cytokine production by human fetal microglia and astrocytes: differential induction by lipopolysaccharide and IL-1 $\beta$," Journal of Immunology, vol. 150, no. 7, pp. 2659-2667, 1993.

[36] C. C. Chao, S. Hu, T. W. Molitor, E. G. Shaskan, and P. K. Peterson, "Activated microglia mediate neuronal cell injury via a nitric oxide mechanism," Journal of Immunology, vol. 149, no. 8, pp. 2736-2741, 1992.

[37] A. J. Herrera, A. Castaño, J. L. Venero, J. Cano, and A. Machado, "The single intranigral injection of LPS as a new model for studying the selective effects of inflammatory reactions on dopaminergic system," Neurobiology of Disease, vol. 7, no. 4, pp. 429-447, 2000.

[38] X. Lu, G. Bing, and T. Hagg, "Naloxone prevents microgliainduced degeneration of dopaminergic substantia nigra neurons in adult rats," Neuroscience, vol. 97, no. 2, pp. 285-291, 2000.

[39] M. D. C. Hernández-Romero, S. Argüelles, R. F. Villarán et al., "Simvastatin prevents the inflammatory process and the dopaminergic degeneration induced by the intranigral injection of lipopolysaccharide," Journal of Neurochemistry, vol. 105, no. 2, pp. 445-459, 2008.

[40] T. Arimoto, D. Y. Choi, X. Lu et al., "Interleukin-10 protects against inflammation-mediated degeneration of dopaminergic neurons in substantia nigra," Neurobiology of Aging, vol. 28, no. 6, pp. 894-906, 2007.

[41] P. F. Hsieh, L. G. Chia, D. R. Ni et al., "Behavior, neurochemistry and histology after intranigral lipopolysaccharide injection," NeuroReport, vol. 13, no. 3, pp. 277-280, 2002.
[42] D. Y. Choi, J. Zhang, and G. Bing, "Aging enhances the neuroinflammatory response and $\alpha$-synuclein nitration in rats," Neurobiology of Aging, vol. 31, no. 9, pp. 1649-1653, 2010.

[43] W. Zhang, T. Wang, Z. Pei et al., "Aggregated $\alpha$-synuclein activates microglia: a process leading to disease progression in Parkinson's disease," FASEB Journal, vol. 19, no. 6, pp. 533542, 2005.

[44] R. L. Hunter, B. Cheng, D. Y. Choi et al., "Intrastriatal lipopolysaccharide injection induces Parkinsonism in C57/B6 mice," Journal of Neuroscience Research, vol. 87, no. 8, pp. 1913-1921, 2009.

[45] R. L. Hunter, N. Dragicevic, K. Seifert et al., "Inflammation induces mitochondrial dysfunction and dopaminergic neurodegeneration in the nigrostriatal system," Journal of Neurochemistry, vol. 100, no. 5, pp. 1375-1386, 2007.

[46] R. L. Hunter, D. Y. Choi, J. F. Kincer, W. A. Cass, G. Bing, and D. M. Gash, "Fenbendazole treatment may influence lipopolysaccharide effects in rat brain," Comparative Medicine, vol. 57, no. 5, pp. 487-492, 2007.

[47] U. Bickel, B. Grave, Y. S. Kang, A. Del Rey, and K. Voigt, "No increase in blood-brain barrier permeability after intraperitoneal injection of endotoxin in the rat," Journal of Neuroimmunology, vol. 85, no. 2, pp. 131-136, 1998.

[48] W. Pan and A. J. Kastin, "TNF $\alpha$ transport across the bloodbrain barrier is abolished in receptor knockout mice," Experimental Neurology, vol. 174, no. 2, pp. 193-200, 2002.

[49] W. A. Banks, "Blood-brain barrier transport of cytokines: a mechanism for neuropathology," Current Pharmaceutical Design, vol. 11, no. 8, pp. 973-984, 2005.

[50] S. L. Byler, G. W. Boehm, J. D. Karp et al., "Systemic lipopolysaccharide plus MPTP as a model of dopamine loss and gait instability in C57Bl/6J mice," Behavioural Brain Research, vol. 198, no. 2, pp. 434-439, 2009.

[51] P. L. McGeer, C. Schwab, A. Parent, and D. Doudet, "Presence of reactive microglia in monkey substantia nigra years after 1methyl-4-phenyl-1,2,3,6-tetrahydropyridine administration," Annals of Neurology, vol. 54, no. 5, pp. 599-604, 2003.

[52] Y. Ouchi, E. Yoshikawa, Y. Sekine et al., "Microglial activation and dopamine terminal loss in early Parkinson's disease," Annals of Neurology, vol. 57, no. 2, pp. 168-175, 2005.

[53] B. Liu, J. W. Jiang, B. C. Wilson et al., "Systemic infusion of naloxone reduces degeneration of rat substantia nigral dopaminergic neurons induced by intranigral injection of lipopolysaccharide," Journal of Pharmacology and Experimental Therapeutics, vol. 295, no. 1, pp. 125-132, 2000.

[54] L. Qian, M. L. Block, S. J. Wei et al., "Interleukin-10 protects lipopolysaccharide-induced neurotoxicity in primary midbrain cultures by inhibiting the function of NADPH oxidase," Journal of Pharmacology and Experimental Therapeutics, vol. 319, no. 1, pp. 44-52, 2006.

[55] R. L. Hunter, D. Y. Choi, S. A. Ross, and G. Bing, "Protective properties afforded by pioglitazone against intrastriatal LPS in Sprague-Dawley rats," Neuroscience Letters, vol. 432, no. 3, pp. 198-201, 2008.

[56] B. Xing, M. Liu, and G. Bing, "Neuroprotection with pioglitazone against LPS insult on dopaminergic neurons may be associated with its inhibition of NF- $\kappa$ B and JNK activation and suppression of COX-2 activity," Journal of Neuroimmunology, vol. 192, no. 1-2, pp. 89-98, 2007.

[57] M. Tomás-Camardiel, I. Rite, A. J. Herrera et al., "Minocycline reduces the lipopolysaccharide-induced inflammatory reaction, peroxynitrite-mediated nitration of proteins, disruption 
of the blood-brain barrier, and damage in the nigral dopaminergic system," Neurobiology of Disease, vol. 16, no. 1, pp. 190201, 2004.

[58] L. W. Fan, YI. Pang, S. Lin et al., "Minocycline reduces lipopolysaccharide-induced neurological dysfunction and brain injury in the neonatal rat," Journal of Neuroscience Research, vol. 82, no. 1, pp. 71-82, 2005.

[59] S. M. Lee, T. Y. Yune, S. J. Kim et al., "Minocycline inhibits apoptotic cell death via attenuation of TNF- $\alpha$ expression following iNOS/NO induction by lipopolysaccharide in neuron/glia co-cultures," Journal of Neurochemistry, vol. 91, no. 3, pp. 568-578, 2004.

[60] B. Ravina, "A randomized, double-blind, futility clinical trial of creatine and minocycline in early Parkinson disease," Neurology, vol. 66, no. 5, pp. 664-671, 2006.

[61] K. Kieburtz, B. Tilley, B. Ravina et al., "A pilot clinical trial of creatine and minocycline in early Parkinson disease: 18month results," Clinical Neuropharmacology, vol. 31, no. 3, pp. 141-150, 2008.

[62] M. O. Griffin, E. Fricovsky, G. Ceballos, and F. Villarreal, "Tetracyclines: a pleitropic family of compounds with promising therapeutic properties. Review of the literature," American Journal of Physiology, vol. 299, no. 3, pp. C539-C548, 2010.

[63] B. Xing, T. Xin, R. L. Hunter, and G. Bing, "Pioglitazone inhibition of lipopolysaccharide-induced nitric oxide synthase is associated with altered activity of p38 MAP kinase and PI3K/Akt," Journal of Neuroinflammation, vol. 5, article 4, 2008.

[64] L. Zecca, H. Wilms, S. Geick et al., "Human neuromelanin induces neuroinflammation and neurodegeneration in the rat substantia nigra: implications for Parkinson's disease," Acta Neuropathologica, vol. 116, no. 1, pp. 47-55, 2008.

[65] H. M. Gao, P. T. Kotzbauer, K. Uryu, S. Leight, J. Q. Trojanowski, and V. M. Y. Lee, "Neuroinflammation and oxidation/nitration of $\alpha$-synuclein linked to dopaminergic neurodegeneration," Journal of Neuroscience, vol. 28, no. 30, pp. 7687-7698, 2008.

[66] L. Zecca, A. Stroppolo, A. Gatti et al., "The role of iron and molecules in the neuronal vulnerability of locus coeruleus and substantia nigra during aging," Proceedings of the National Academy of Sciences of the United States of America, vol. 101, no. 26, pp. 9843-9848, 2004.

[67] M. Mittelbronn, K. Dietz, H. J. Schluesener, and R. Meyermann, "Local distribution of microglia in the normal adult human central nervous system differs by up to one order of magnitude," Acta Neuropathologica, vol. 101, no. 3, pp. 249255,2001

[68] L. J. Lawson, V. H. Perry, P. Dri, and S. Gordon, "Heterogeneity in the distribution and morphology of microglia in the normal adult mouse brain," Neuroscience, vol. 39, no. 1, pp. 151-170, 1990.

[69] J. Miklossy, "Chronic inflammation and amyloidogenesis in Alzheimer's disease-role of spirochetes," Journal of Alzheimer's Disease, vol. 13, no. 4, pp. 381-391, 2008.

[70] A. D. Roth, G. Ramírez, R. Alarcón, and R. von Bernhardi, "Oligodendrocytes damage in Alzheimer's disease: beta amyloid toxicity and inflammation," Biological Research, vol. 38, no. 4, pp. 381-387, 2005. 


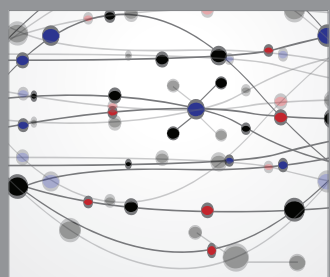

The Scientific World Journal
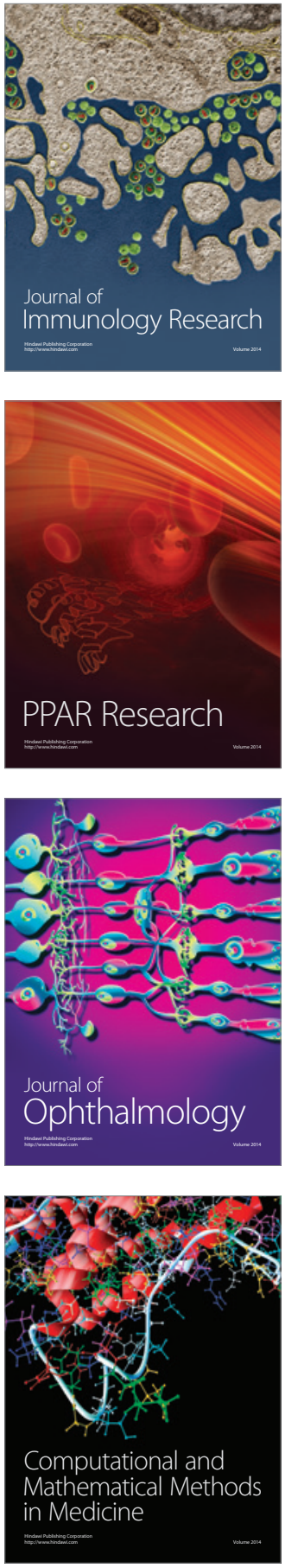

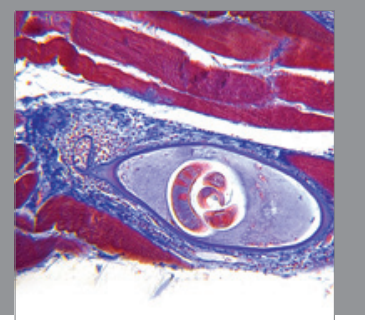

Gastroenterology

Research and Practice
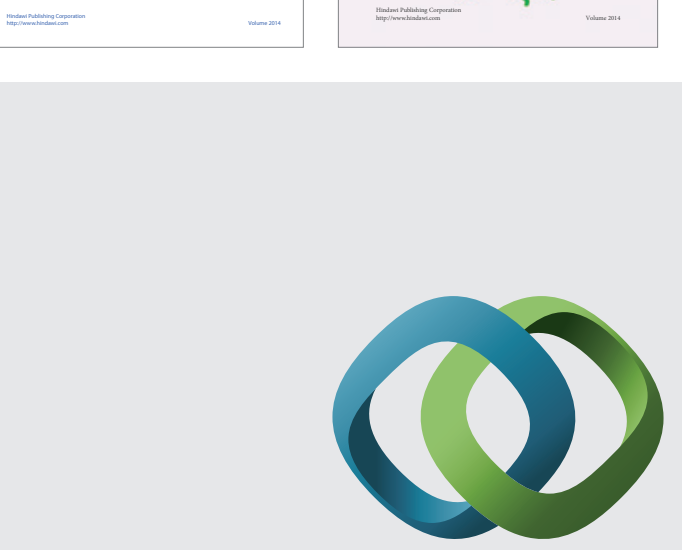

\section{Hindawi}

Submit your manuscripts at

http://www.hindawi.com
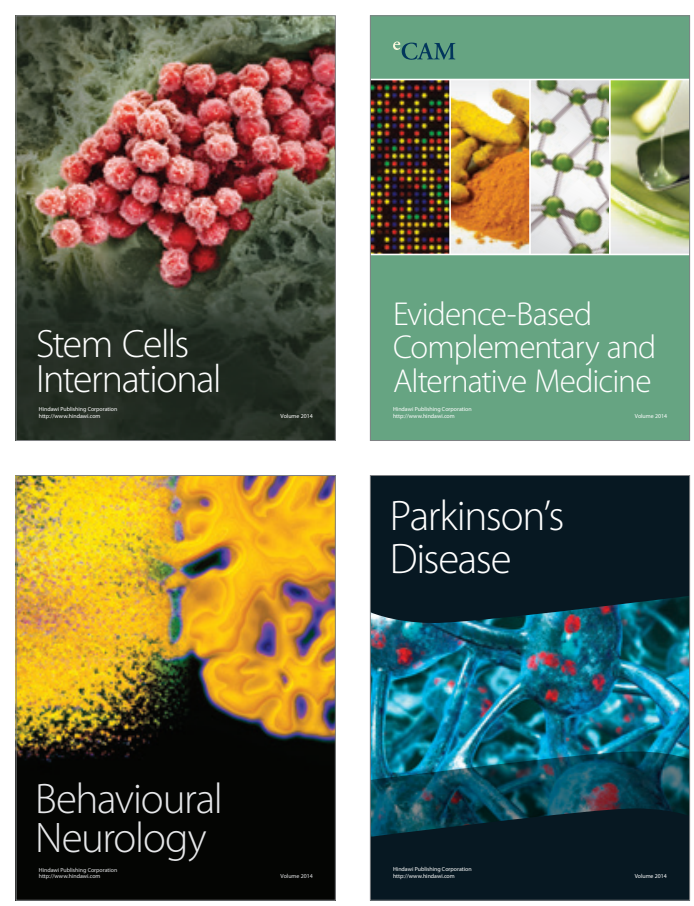

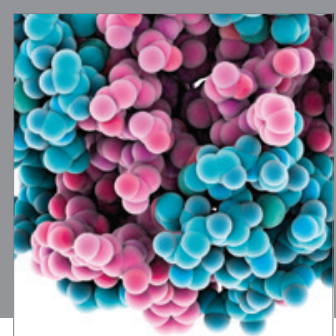

Journal of
Diabetes Research

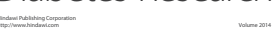

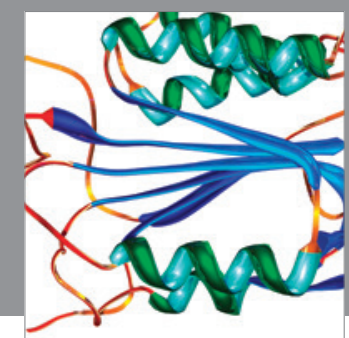

Disease Markers
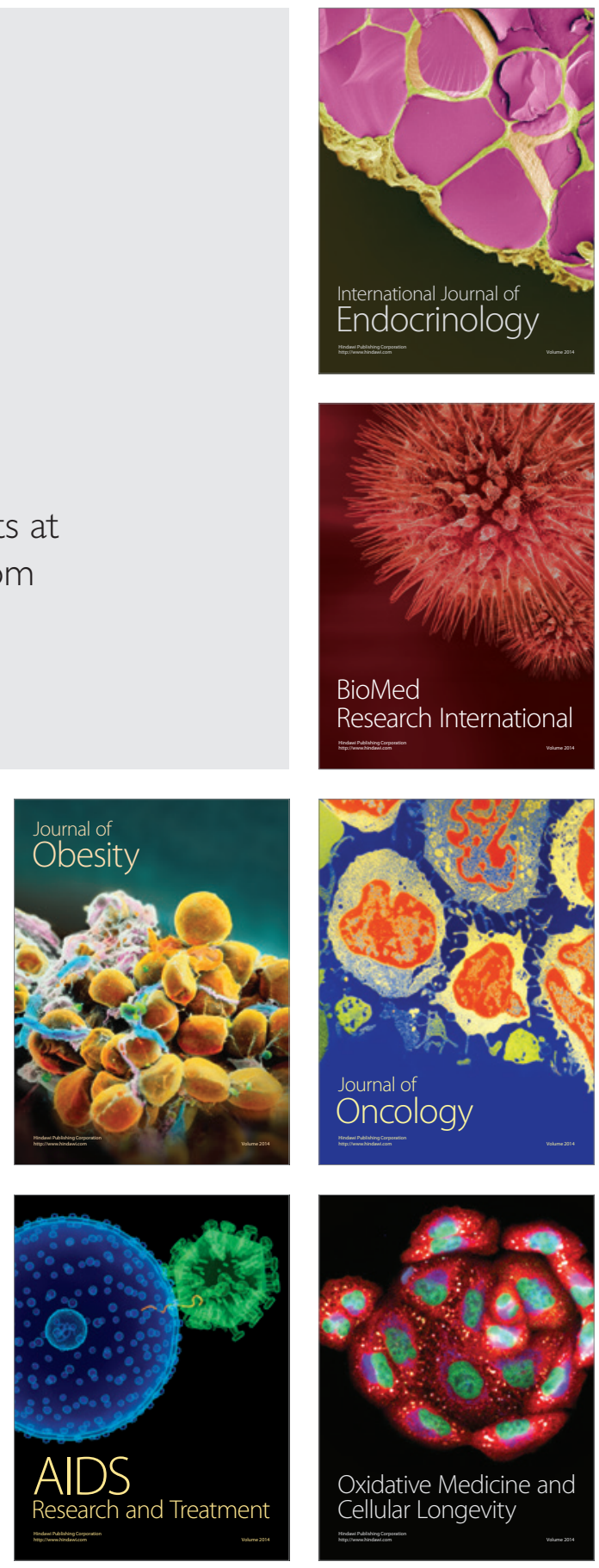